DOI: 10.12731/wsd-2018-2-27-39

УДК 612.766:616-053.9

\title{
ХАРАКТЕРИСТИКА ПАРАМЕТРОВ ХОДЬБЫ У ЖЕНЩИН ПОЖИЛОГО ВОЗРАСТА ПРИ ИЗБЫТОЧНОЙ МАССЕ ТЕЛА
}

\author{
Мороз Т.П., Федотов Д.М., Попова О.Н., \\ Пащенко В.П., Грибанов А.В.
}

Цель. Выявить особенности ходьбы у женщин пожилого возраста с избыточной массой тела (MT).

Методы. В исследовании приняли участие 323 женщины в возрасте 55-74 лет. Измерение длинь тела (ДТ) и массы тела (МТ) производилось по стандартной методике. Расчет индекса МТ (ИМТ) производился по формуле: МТ (кг) ДТ (см). В первую группу - группу исследования (ГИ) - вошли женщины, с избыточной $M T(n=162)$. Во вторую группу группу сравнения (ГС) - женщиины, с нормальной МТ $(n=161)$. Особенности ходьбы изучали с помощью следующих тестов компьютерного стабилометрического комплекса «Balance Manager»: 1) Вставание из положения сидя; 2) Простая ходьба; 3) Тандемная ходьба. Статистическая обработка результатов проводилась с использованием пакета программ «SPSS 23.0».

Результаты. У пожилых женщин с избытком МТ отмечается значимое снижение величины индекса подъема на 13,8\% (p=0,020) и возрастание скорости колебания ЦТ на 12,8\% (p=0,002). Также в ГИ установлено снижение длины шага на 6\% $(p=0,015)$ и скорости ходьбы на $3,8 \%(p=0,023)$ с одновременным возрастанием ширины шага (на 9,4\% $(p=0,001)$,) могут рассматриваться как признаки адаптивных перестроек параметров простой ходьбы при увеличении массы тела.

Заключение. Таким образом, при оченке пространственно-временных характеристик движения у женщин 55-74 лет в зависимости от ИМТ нами выявлень изменения, свидетельствующие об ухудшении функционирования нейрофизиологических механизмов обеспечивающих удержание вертикальной позы.

Ключевые слова: постуральный баланс; пожилой возраст; женщины избыток массы тела. 


\title{
CHARACTERISTIC OF WALK PARAMETERS IN ELDERLY WOMEN WITH OVERWEIGHT
}

\author{
Moroz T.P., Fedotov D.M., Popova O.N., \\ Pashenko V.P., Gribanov A.V.
}

Background: Identify the features of walking in elderly women with overweight.

Materials and Methods: The study involved 109 women aged 55-74. Measurement of length and body weight was performed according to a standard procedure. Calculation of the BMI was made by formula: Body weight (kg) / body length ${ }^{2}(\mathrm{~cm})$. The first group - study group (SG) included women with overweight $(n=162)$. The second group - comparison group $(C G)$ were taken women with normal body weight $(n=161)$. Features of walking were studied using the following tests of the computer stabilometric complex "Balance Manager": 1) Sit-to-stand test; 2) Walk Across; 3) Tandem Walk. The statistical processing of the results was carried out using the "SPSS 23.0" software package. The critical level of significance ( $p$ ) in testing the statistical hypotheses in the study was $p \leq 0.05$.

Results: There were a significant decrease in the rising index by $13.8 \%$ $(p=0.020)$ and an increase in the sway velocity by $12.8 \%(p=0.002)$ in elderly women with overweight. Also in the $S G$, a decrease in the step length by $6 \%(p=0.015)$ and speed of walking of $3.8 \%(p=0.023)$ with a simultaneous increasing in the step width by $9.4 \%(p=0.001)$ can be considered as signs adaptive rearrangements of simple walk parameters with increasing of body weight.

Conclusion: Thus, while assessing the spatial-temporal characteristics of motion in women aged 55-74, depending on the BMI, we detected changes that indicate deterioration in the functioning of the neurophysiological mechanisms that provide keeping of the vertical posture.

Keywords: postural balance; elderly age; women; overweight.

В настоящее время увеличение в человеческой популяции лиц с избытком массы тела (МТ) становится одной из наиболее острых проблем здравоохранения. Доказано, что у женщин пожилого возраста избыток МТ является предиктором инвалидизации, при этом данный риск сохраняется даже до глубокой старости [1, с. 1093-1098]. 
Накопление избытка МТ вызывает множественные физиологические перестройки в организме человека, в значительной степени снижая скоординированность работы суставов и мышц, ответственных за поддержание постурального баланса. Лица с более высокой МТ демонстрируют трудности при сгибании, вставании на колени, вставании из различных положений, стоянии и ходьбе. При избытке МТ отмечается увеличение объема и массы жировой ткани в брюшной полости, приводящее к смещению центра массы тела вперед, что вызывает необходимость возрастания нагрузки на голеностопный сустав для поддержания постуральной устойчивости [2, с. 126-136, 3, с. 1750-1757, 4, с. 931-941]. В исследованиях Gilleard [5, с. 267-271], Bertocco [6, с. 131-137] показано, что при избытке МТ снижается способность сохранять и восстанавливать равновесие в связи с ограничением диапазон движения туловища и верхних конечностей. Проблемы, связанные с выполнением этих физических задач создают ограничения подвижности при осуществлении основных видов деятельности в повседневной жизни человека, значительно снижая его социальное функционирование. В свою очередь, поддержание ИМТ ниже 75-го процентиля способствует сохранению физических возможностей и высокого уровня физического функционирования [7, с. 698-702].

Известно, что с возрастом также отмечается снижение постуральной устойчивости, увеличение площади базы опоры, снижение скорости и длины шага, ограничение амплитуды движения суставов, уменьшение управления балансом во фронтальной и сагиттальной плоскостях [8, c. $1-10,9$, с. $265-274,10$, с. $513-520,11$, с. 50-54]. Что в свою очередь может приводить к развитию синдрома падений [12, с. 83-86]. При этом накопление избытка МТ может способствовать интенсификации снижения постуральной устойчивости в пожилом возрасте [13, с. 475-481]. Таким образом, целью данного исследования являлось выявление особенностей ходьбы у женщин пожилого возраста с избыточной массой тела.

\section{Материалы и методы}

Обследовано 323 женщины в возрасте 55-74 лет. Измерение ДТ и МТ производилось с помощью электронного ростомера по стандартной методике. Расчет ИМТ производился по формуле: МТ (кг) / ДТ² (см). Известно, что при оценке ИМТ наиболее распространенной является классификация ВО3: 16,0-18,5 - недостаточная (дефицит) масса тела; 18,5-24,9 норма; ИМТ $\geq 25,0$ - избыточный вес; ИМТ $\geq 30,0$ - ожирение. Однако в связи с тем, что субъектом нашего исследования являлись лица пожи- 
лого возраста, мы использовали классификацию Комитета по питанию и здоровью (США, 1989): $\leq 22,9$ - дефицит массы тела; 23,0-29,0 - норма; $\geq 29,0$ - избыток массы тела. В работе Babiarczyk В. [14, с. 58-67] показано, что эта классификация является наиболее информативной при оценке ИМТ у лиц данной возрастной группы.

В первую группу - группу исследования (ГИ) - вошли женщины, с избыточной МТ (n=162). Во вторую группу - группу сравнения (ГС) - женщины, с нормальной МТ (n=161).

Все женщины на момент обследования были мобильны и не использовали специальные средства для дополнительной опоры при ходьбе. В работу не вошли лица, находящиеся на учете в психоневрологических диспансерах, имеющие в анамнезе инсульты, деменцию и черепно-мозговые травмы.

Особенности ходьбы изучали с помощью следующих тестов компьютерного стабилометрического комплекса «Balance Manager»: 1) вставание из положения сидя - для оценки времени перемещения веса, индекса подъема и скорости колебания центра тяжести (ЦТ) при вставании из положения сидя; 2) простая ходьба - для оценки ширины и длины шага, скорости ходьбы при переходе обследуемым от одного конца платформы к другому; 3) тандемная ходьба - для оценки ширины шага, скорости ходьбы и конечного колебания при ходьбе по прямой линии от одного конца платформы к другому, приставляя как можно ближе носок к пятке.

Статистическая обработка результатов проводилась с использованием пакета программ «SPSS 23.0». В случае нормального распределения результаты представляли в виде среднего значения (M) и стандартного отклонения (s), при ненормальном - в виде медианы (Me), первого (Q1) и третьего (Q3) квартилей. При нормальном распределении количественных данных статистическую значимость различий оценивали с помощью парного t-критерия Стьюдента, при ненормальном - с помощью критерия Вилкоксона. Критический уровень значимости (р) при проверке статистических гипотез в исследовании принимали $\mathrm{p} \leq 0,05$.

\section{Результаты исследование и их обсуждение}

Основные антропометрические характеристики обследованных женщин пожилого возраста представлены в таблице 1. Среди пожилых женщин ГИ отмечается ожидаемо значимое увеличение показателей МТ $(\mathrm{p}<0,001)$ и ИМТ $(\mathrm{p}<0,001)$ по сравнению с ГС. 
Таблий 1.

Характеристика обследованной выборки

\begin{tabular}{|c|c|c|c|}
\hline & ГИ & ГС & Р \\
\hline Возраст & $63,0(60,0-67,0)$ & $64,0(60,0-68,0)$ & 0,117 \\
\hline ДТ, см & $157,5(153,7-161,0)$ & $157,0(154,0-161,0)$ & 0,811 \\
\hline МТ, кг & $82,5(76,0-92,2)$ & $67,0(60,3-71,7)$ & $<0,001$ \\
\hline ИМТ, усл. ед. & $32,9(31,2-35,6)$ & $27,1(25,9-28,6)$ & $<0,001$ \\
\hline
\end{tabular}

При анализе результатов теста «Вставание из положения сидя» статистически значимые различия выявлены в показателях: индекс подъема $(\mathrm{p}=0,020)$ и скорость колебания центра тяжести $(\mathrm{p}=0,002)$ (таблица 2). Известно, что вставание зависит от мышечно-связочного аппарата коленных суставов и требует большей мышечной силы ног, нежели другие двигательные акты, такие как простая или сложная ходьба. Стоит так же заметить, что наблюдается обратная зависимость между величиной индекса подъема и необходимым количеством затрачиваемой мышечной силы. Таким образом, отмечаемое в ГИ снижение величины индекса подъема на 13,8\% и возрастание скорости колебания ЦТ на 12,8\% может свидетельствовать о наличии изменений нейрофизиологических механизмов обеспечивающих удержание вертикальной позы у женщин пожилого возраста с избытком МТ.

При оценке результатов теста «Простая ходьба» установлены статистически значимые различия во всех измеряемых параметрах предложенного теста: ширина шага $(\mathrm{p}=0,001)$, длина шага $(\mathrm{p}=0,015)$ и скорость ходьбы $(\mathrm{p}=0,023)$. Известно, что существует множество факторов, влияющих на скорость ходьбы у лиц пожилого возраста [15, с. 215-221]. При этом МТ рассматривается в качестве одной из наиболее значимых детерминант, влияющих на скорость ходьбы. Отмечаемое в ГИ снижение длины шага (на 6\%) и скорости ходьбы (на 3,8 \%) с одновременным возрастанием ширины шага (на 9,4\%) могут рассматриваться как признаки адаптивных перестроек параметров простой ходьбы при увеличении массы тела. Известно, что на скорость простой ходьбы и ее снижение могут оказывать значительное влияние такие факторы, как состояние здоровья, избыток МТ и низкая физическая активность [16, с. 715-720]. В исследовании Stenholm S c соавт. [17, с. 277-283] показано, что избыток МТ значимо увеличивает риск ухудшения показателей ходьбы, вне зависимости от заболеваний, вызванных ожирением, курением, семейным положением и образованием, особенно у пожилых женщин. Также установлено, 
что у лиц с избытком массы тела отмечаются более высокие энергетические затраты на ходьбу [18 с. 1017-1025]. Таким образом, ухудшение показателей теста «Простая ходьба» также может рассматриваться как индикатор снижения физической работоспособности у женщин пожилого возраста с избытком МТ [19, с. 170-175].

Таблийа 2.

Параметры постурального баланса у женщин пожилого возраста

\begin{tabular}{|c|c|c|c|c|}
\hline \multirow[b]{2}{*}{ Тест } & \multirow[b]{2}{*}{ Показатель } & \multicolumn{2}{|c|}{ ИМТ } & \multirow[b]{2}{*}{$\mathrm{p}$} \\
\hline & & $\begin{array}{c}\text { ГИ } \\
\mathrm{n}=162\end{array}$ & $\begin{array}{c}\Gamma C \\
n=161\end{array}$ & \\
\hline \multirow{3}{*}{$\begin{array}{c}\text { Вставание из } \\
\text { положения } \\
\text { сидя }\end{array}$} & $\begin{array}{c}\text { Время перемещения } \\
\text { веса, с }\end{array}$ & $\begin{array}{c}0,45 \\
(0,32-0,55)\end{array}$ & $\begin{array}{c}0,47 \\
(0,36-0,60) \\
\end{array}$ & 0,143 \\
\hline & $\begin{array}{c}\text { Индекс подъема, \% от } \\
\text { МТ }\end{array}$ & $\begin{array}{c}15,5 \\
(12,00-20,00)\end{array}$ & $\begin{array}{c}18,00 \\
(14,00-22,00)\end{array}$ & $0,020^{*}$ \\
\hline & $\begin{array}{c}\text { Скорость колебания } \\
\text { ЦТ, град/с }\end{array}$ & $\begin{array}{c}3,80 \\
(3,16-4,50) \\
\end{array}$ & $\begin{array}{c}3,37 \\
(2,73-4,15) \\
\end{array}$ & $0,002 * *$ \\
\hline \multirow{3}{*}{$\begin{array}{c}\text { Простая } \\
\text { ходьба }\end{array}$} & Ширина шага, см & $13,73 \pm 3,04$ & $12,55 \pm 3,05$ & $0,001 * * *$ \\
\hline & Длина шага, см & $\begin{array}{c}54,80 \\
(49,57-61,58) \\
\end{array}$ & $\begin{array}{c}58,30 \\
(52,55-62,46) \\
\end{array}$ & $0,015^{*}$ \\
\hline & Скорость ходьбы, см/с & $82,48 \pm 13,20$ & $85,75 \pm 12,40$ & $0,023 *$ \\
\hline \multirow{3}{*}{$\begin{array}{c}\text { Тандемная } \\
\text { ходьба }\end{array}$} & Ширина шага, см & $\begin{array}{c}7,43 \\
(6,50-9,32)\end{array}$ & $\begin{array}{c}7,20 \\
(6,25-8,75)\end{array}$ & 0,270 \\
\hline & Скорость, см/с & $\begin{array}{c}28,48 \\
(22,90-34,20) \\
\end{array}$ & $\begin{array}{c}29,30 \\
(23,65-36,19) \\
\end{array}$ & 0,193 \\
\hline & $\begin{array}{c}\text { Конечное колебание, } \\
\text { град/с }\end{array}$ & $\begin{array}{c}5,30 \\
(4,56-6,60)\end{array}$ & $\begin{array}{c}5,30 \\
(3,96-6,80)\end{array}$ & 0,432 \\
\hline
\end{tabular}

Примечание: $\mathrm{p}^{*}$ - статистически значимые различия.

Также нами был проведен тест «Тандемная ходьба», однако анализ его результатов не выявил статистически значимых различий. Соответственно, не установлено изменение нейрофизиологических механизмов, обеспечивающих сложно-координационные движения в сравниваемых группах, и не выявлено снижение адаптационных способностей к сложным двигательным актам.

Известно, что реализация мероприятий, направленных на сохранение двигательной активности, способности к выполнению сложных двигательных актов у пожилых людей благоприятно влияет на качество жизни, позволяя снизить риск последующих падений и сохранить социальную активность [20, с. 300-309, 21, с. 44-52]. При этом в использова- 
ние тестов «Простая ходьба» и «Вставание из положения сидя» позволит оценить эффективность программ, направленных на сохранение мобильности у пожилых людей.

\section{Заключение}

Таким образом, избыточная масса тела у женщин пожилого возраста способствует ухудшению функционирования нейрофизиологических механизмов обеспечивающих удержание вертикальной позы при вставании из положения сидя и оказывает отрицательное воздействие на пространственно-временных характеристики движения. В свою очередь значимое снижение длины шага, скорости ходьбы и увеличение ширины шага у женщин с избытком МТ могут рассматриваться в качестве индикаторов процесса адаптивных перестроек параметров простой ходьбы. Использование тестов «Простая ходьба» и «Вставание из положения сидя» при диагностике параметров ходьбы у пожилых женщин с избытком массы тела может найти свое применение в прогнозировании риска падений и снижения мобильности, а также для разработки и оценки качества коррекционно-реабилитационных программ, связанных с изменениями постурального контроля у пожилых женщин с избытком МТ.

Исследование выполнено при финансовой поддержке РГНФ и администрации Архангельской области в рамках регионального конкурса «Русский Север: история, современность, перспективы», проект № 1716-29003 «Нарушения постурального контроля при старении как фактор снижения качества жизни населения в ичиркумполярных условиях».

\section{Список литературы}

1. Launer L.J.,Harris T., Rumpel C., Madans J. Body Mass Index, Weight Change, and Risk of Mobility Disability in Middle-aged and Older Women The Epidemiologic Follow-up Study of NHANES I. // Journal American Medical Associacion, 1997, vol. 271, no 14, pp. 1093-1098.

2. Corbeil P., Simoneau M., Rancourt D., Tremblav A., Teamblav N. Increased risk for falling associated with obesity: mathematical modeling of postural control // IEEE Transactions on Neural Systems and Rehabilitation Engineering, 2001, vol. 9, no. 2, pp. 126-136.

3. Berrigan F.F., Simoneau M.M., Tremblay A.A., Hue O.O., Teasdale N.N. Influence of obesity on accurate and rapid arm movement performed from standing posture // International Journal of Obesity, 2006, vol. 30, no. 12, pp. 1750-1757. 
4. Li X., Aruin A.S. The effect of short-term changes in body mass distribution on feed-forward postural control // Journal of Electromyography \& Kinesiology, 2009, vol. 19, no. 5, pp. 931-941.

5. Gilleard W.W., Smith T.T. Effect of obesity on posture and hip joint moments during a standing task, and trunk forward flexion motion // International Journal of Obesity, 2007, vol. 31, no. 2, pp. 267-271.

6. Bertocco P., Baccalaro G., Montesano A., Vismara L., Parisio C., Galli M., The analysis of sit-to-stand movement in obese and normal subjects: Biomechanic evaluations and postural changes between groups // Europa Medicophysica, 2002, vol. 38, no. 3, pp. 131-137.

7. Harris T., Kovar M.G., Suzman R., Kleinman J.C., Feldman J.J. Longitudinal Study of Physical Ability in the Oldest-Old // The American Journal of Public Health, 1989, vol. 79, no. 6, pp. 698-702.

8. Шварц Г.Я. Остеопороз, падения и переломы в пожилом возрасте: роль D-эндокринной системы // Русский Медицинский Журнал. 2008. Том 16. № 10. C. 1-10.

9. Axer H., Axer M., Sauer H. Falls and gait disorders in geriatric neurology // Clinical Neurology and Neurosurgery, 2010, vol. 112, pp. 265-274.

10. Гудков А.Б., Дёмин А.В. Особенности постурального баланса у мужчин пожилого и старческого возраста с синдромом страха падения // Успехи геронтологии. 2012. Т. 25. № 1. С. 166-170.

11. Демин А.В., Гудков А.Б., Грибанов А.В. Особенности постуральной стабильности у мужчин пожилого и старческого возраста // Экология человека. 2010. № 12. С. 50-54.

12. Мороз Т.П. Возрастные особенности параметров ходьбы у женщин 55-64 лет, проживающих в условиях Северо-Арктического региона // Вестник Уральской медицинской академической науки. 2014. № 2 (48). С. 83-86.

13. Carneiro J.A.O., Santos-Pontelli T.E.G., Vilaca K.H.C., Pfrimer K., Colafemina J.F., Carneiro A.A.O., Ferriolli E. Obese elderly women exhibit low postural stability: a novel three-dimensional evaluation system // Clinics, 2012, vol. 67, no. 5, pp. 475-481.

14. Babiarczyk B., Turbiarz A. Body Mass Index in elderly people - do the reference ranges matter? // Progress in Health Sciences, 2011, vol. 1, no. 2, pp. 58-67.

15. Inoue W., Ikezoe T., Tsuboyama T., Sato I., Malinowska K.B., Kawaguchi T., Tabara Y., Nakayama T., Matsuda F., Ichihashi N. Are there different factors affecting walking speed and gait cycle variability between men and women in community-dwelling older adults? // Aging Clinical and Experimental Research, 2017, vol. 29, no. 2, pp. 215-221. 
16. Newman A.B., Haggerty C.L., Kritchevsky S.B. et al. Walking Performance and Cardiovascular Response: Associations with Age and Morbidity - the Health, Aging and Body Composition Study // The Journals of Gerontology. Series A: Biological Sciences and Medical Science, 2003, vol. 58, no. 8, pp. 715-720.

17. Stenholm S., Sainio P., Rantanen T., Alanen E., Koskinen S. Effect of co-morbidity on the association of high body mass index with walking limitation among men and women aged 55 years and older // Aging clinical and experimental research, 2007, no. 19, pp. 277-283.

18. Laroche D.P., Marques N.R., Shumila H.N., Logan C.R., Laurent R.S. Excess Body Weight and Gait Influence Energy Cost of Walking in Older Adults // Medicine \& Science in Sports \& Exercise, 2015 vol. 47, no. 5, pp. 1017-1025.

19. Alipanah N., Varadhan R., Sun K., Ferrucci L., Fried L.P., Semba R.D. Low Serum Carotenoids Are Associated with a Decline in Walking Speed in Older Women // The Journal of Nutrition, Health \& Aging, 2009. vol. 13, no. 3, pp. 170-175.

20. Beyer N., Simonsen L., Bülow J., Lorenzen T., Jensen D.V., Larsen L., Rasmussen U., Rennie M., Kjaer M. Old Women with a Recent Fall History Show Improved Muscle Strength and Function Sustained for Six Months After Finishing Training // Aging Clinical and Experimental Research, 2007. Vol. 19, № 4, pp. 300-309.

21. Мелькова Л.А., Федотов Д.М. Состояние вегетативной регуляции ритма сердца при пассивном ортостазе у женщин пожилого и старческого возраста // Журнал медико-биологических исследований. 2015. № 2. С. 44-52.

\section{References}

1. Launer L.J.,Harris T., Rumpel C., Madans J. Body Mass Index, Weight Change, and Risk of Mobility Disability in Middle-aged and Older Women The Epidemiologic Follow-up Study of NHANES I. Journal American Medical Associacion, 1997, vol. 271, no 14, pp. 1093-1098.

2. Corbeil P., Simoneau M., Rancourt D., Tremblav A., Teamblav N. Increased risk for falling associated with obesity: mathematical modeling of postural control. IEEE Transactions on Neural Systems and Rehabilitation Engineering, 2001, vol. 9, no. 2, pp. 126-136.

3. Berrigan F.F., Simoneau M.M., Tremblay A.A., Hue O.O., Teasdale N.N. Influence of obesity on accurate and rapid arm movement performed from standing posture. International Journal of Obesity, 2006, vol. 30, no. 12, pp. 1750-1757.

4. Li X., Aruin A.S. The effect of short-term changes in body mass distribution on feed-forward postural control. Journal of Electromyography \& Kinesiology, 2009, vol. 19, no. 5, pp. 931-941. 
5. Gilleard W.W., Smith T.T. Effect of obesity on posture and hip joint moments during a standing task, and trunk forward flexion motion. International Journal of Obesity, 2007, vol. 31, no. 2, pp. 267-271.

6. Bertocco P., Baccalaro G., Montesano A., Vismara L., Parisio C., Galli M., The analysis of sit-to-stand movement in obese and normal subjects: Biomechanic evaluations and postural changes between groups. Europa Medicophysica, 2002, vol. 38, no. 3, pp. 131-137.

7. Harris T., Kovar M.G., Suzman R., Kleinman J.C., Feldman J.J. Longitudinal Study of Physical Ability in the Oldest-Old. The American Journal of Public Health, 1989, vol. 79, no. 6, pp. 698-702.

8. Shvarc G.Ja. Osteoporoz, padenija i perelomy v pozhilom vozraste: rol' D-jendokrinnoj sistemy [Osteoporosis, falls and fractures in elderly: the role of the D-endocrine system]. Russkij Medicinskij Zhurnal [Russian Medical Journal]. 2008. Vol. 16. № 10, pp. 1-10.

9. Axer H., Axer M., Sauer H. Falls and gait disorders in geriatric neurology. Clinical Neurology and Neurosurgery, 2010, vol. 112, pp. 265-274.

10. Gudkov A.B., Dyomin A.V. Osobennosti postural'nogo balansa u muzhchin pozhilogo i starcheskogo vozrasta s sindromom strakha padeniya [Peculiarities of postural balance among elderly men with fear of falling syndrome]. Uspehi gerontologii [Advantages in Gerontology]. 2012. Vol. 25. № 1, pp. 166-170.

11. Demin A.V., Gudkov A.B., Gribanov A.V. Osobennosti postural'noy stabil'nosti u muzhchin pozhilogo i starcheskogo vozrasta [Features of postural balance in elderly and old man]. Jekologija cheloveka [Human Ecology]. 2010. № 12, pp. 50-54.

12. Moroz T.P. Vozrastnye osobennosti parametrov hod'by u zhenshchin 55-64 let, prozhivayushchih $\mathrm{v}$ usloviyah Severo-Arkticheskogo regiona [Features of walk in women 55-64 years living in Arctic Conditions]. Vestnik Ural'skoj medicinskoj akademicheskoj nauki [Journal of Ural Medical Academic Science]. 2014. № 2 (48), pp. 83-86.

13. Carneiro J.A.O., Santos-Pontelli T.E.G., Vilaca K.H.C., Pfrimer K., Colafemina J.F., Carneiro A.A.O., Ferriolli E. Obese elderly women exhibit low postural stability: a novel three-dimensional evaluation system. Clinics, 2012, vol. 67, no. 5, pp. 475-481.

14. Babiarczyk B., Turbiarz A. Body Mass Index in elderly people - do the reference ranges matter? Progress in Health Sciences, 2011, vol. 1, no. 2, pp. 58-67.

15. Inoue W., Ikezoe T., Tsuboyama T., Sato I., Malinowska K.B., Kawaguchi T., Tabara Y., Nakayama T., Matsuda F., Ichihashi N. Are there different factors affecting walking speed and gait cycle variability between men and women in community-dwelling older adults? Aging Clinical and Experimental Research, 2017, vol. 29, no. 2, pp. 215-221. 
16. Newman A.B., Haggerty C.L., Kritchevsky S.B. et al. Walking Performance and Cardiovascular Response: Associations with Age and Morbidity - the Health, Aging and Body Composition Study. The Journals of Gerontology. Series A: Biological Sciences and Medical Science, 2003, vol. 58, no. 8, pp. 715-720.

17. Stenholm S., Sainio P., Rantanen T., Alanen E., Koskinen S. Effect of co-morbidity on the association of high body mass index with walking limitation among men and women aged 55 years and older. Aging clinical and experimental research, 2007, no. 19, pp. 277-283.

18. Laroche D.P., Marques N.R., Shumila H.N., Logan C.R., Laurent R.S. Excess Body Weight and Gait Influence Energy Cost of Walking in Older Adults. Medicine \& Science in Sports \& Exercise, 2015 vol. 47, no. 5, pp. 1017-1025.

19. Alipanah N., Varadhan R., Sun K., Ferrucci L., Fried L.P., Semba R.D. Low Serum Carotenoids Are Associated with a Decline in Walking Speed in Older Women. The Journal of Nutrition, Health \& Aging, 2009. vol. 13, no. 3, pp. 170-175.

20. Beyer N., Simonsen L., Bülow J., Lorenzen T., Jensen D.V., Larsen L., Rasmussen U., Rennie M., Kjaer M. Old Women with a Recent Fall History Show Improved Muscle Strength and Function Sustained for Six Months After Finishing Training. Aging Clinical and Experimental Research, 2007. Vol. 19, № 4, pp. 300-309.

21. Melkova L.A., Fedotov D.M. Sostoyanie vegetativnoy regulyatsii ritma serdtsa pri passivnom ortostaze $u$ zhenshchin pozhilogo i starcheskogo vozrasta [The state of vegetative regulation of heart rhythm with passive orthostasis in elderly and senile women]. Zhurnal mediko-biologicheskikh issledovaniy [Journal of Medical Biological Research]. 2015. № 2, pp. 44-52.

\section{ДАННЫЕ ОБ АВТОРАХ}

Мороз Таисия Петровна, младший научный сотрудник института медико-биологических исследований

Северньй (Арктический) федеральньий университет имени М.В. Ломоносова

ул. Бадигина, 3, г. Архангельск, 163000, Российская Федерация t.moroz@narfu.ru

Федотов Денис Михайлович, кандидат медицинских наук, доцент кафедры гигиены и медицинской экологии, магистрант 2 курса Высшей школы естественных наук и технологий САФУ

Северный государственный медицинский университет 
пр-т. Троицкий,51, г. Архангельск, 163000, Российская Федерация doctorpro@yandex.ru

Попова Ольга Николаевна, доктор медицинских наук, доцент, профессор кафедры гигиены и медицинской экологии Северный государственный медицинский университет пр-т. Троиџкий,51, г. Архангельск, 163000, Российская Федерация

Пащенко Владимир Петрович, доктор медицинских наук, профессор, профессор кафедры нормальной физиологии Северный государственный медицинский университет пр-т. Трочикий,51, г. Архангельск, 163000, Российская Федерация

Грибанов Анатолий Владимирович, доктор медицинских наук, профессор, главный научный сотрудник института медико-биологических исследований

Северный (Арктический) федеральный университет имени М.В. Ломоносова

ул. Бадигина, 3, г. Архангельск, 163000, Российская Федерация

\section{DATA ABOUT THE AUTHORS}

Moroz Taisiia Petrovna, Junior Researcher, Institute of Biomedical Research Northern (Arctic) Federal University

3, Badigina Str., Arkhangelsk, 163000, Russian Federation t.moroz@narfu.ru

Fedotov Denis Mikhailovich, $\mathrm{PhD}$, Associate Professor of the Department of Hygiene and Medical Ecology; Master student of Higher School of Natural Sciences and Technology, Northern (Arctic) Federal University.

Northern State Medical University

51, Troitsky av., Arkhangelsk, 163000, Russian Federation doctorpro@yandex.ru

Popova Olga Nikolaevna, Doctor of Medical Sciences, Associate Professor, Professor of the Department of Hygiene and Medical Ecology Northern State Medical University

51, Troitsky av., Arkhangelsk, 163000, Russian Federation 
Pashchenko Vladimir Petrovich, Doctor of Medical Sciences, Professor, Professor of the Department of Department of Normal Physiology

Northern State Medical University

51, Troitsky av., Arkhangelsk, 163000, Russian Federation

Gribanov Anatoly Vladimirovich, Doctor of Medical Sciences, Professor, Chief researcher, Institute of Biomedical Research

Northern (Arctic) Federal University

3, Badigina Str., Arkhangelsk, 163000, Russian Federation 